\title{
SAPIEN 3 Ultra - Design and procedural features of a new balloon-expandable valve
}

\author{
Radosław Parma ${ }^{1 *}$, Damian Hudziak ${ }^{2 *}$, Grzegorz Smolka ${ }^{1}$, \\ Radosław Gocol ${ }^{2}$, Andrzej Ochała ${ }^{1}$, Wojciech Wojakowski ${ }^{1}$ \\ ${ }^{1}$ Department of Cardiology and Structural Heart Diseases, \\ Medical University of Silesia, Katowice, Poland \\ ${ }^{2}$ Department of Cardiac Surgery, Medical University of Silesia, Katowice, Poland
}

\section{Introduction}

Balloon-expandable (BE) SAPIEN 3 transcatheter heart valves (Edwards Lifesciences, Irvine, CA, USA) have a track record of excellent deliverability, low risk of paravalvular regurgitation (PVL), permanent pacemaker implantation and incidence of stroke. This technology evolved into the current version by reduction of the delivery profile achieved by alignment of the valve on the balloon in the descending aorta, improvement of sealing by the outer PET skirt and use of an expandable, hydrophilic-coated femoral sheath. In February 2019 a new system - SAPIEN 3 Ultra was launched in Poland. It has important new features which warrant discussion: 1) higher outer skirt with redesigned structure, 2) delivery system (low profile nosecone, on-balloon valve crimping, dual articulation), 3 ) expandable, seamless delivery sheath. The valve $\mathrm{CoCr}$ stent, bovine pericardial leaflets, and inner sealing skirt remain unchanged (Fig. 1).

The main goals for the design improvements are simplification of the procedure, further reduction of PVL risk and a decrease in vascular complications [1].

\section{The redesigned outer sealing skirt}

Results of the PARTNER trials showed a very low risk of PVL, in particular when using the $29 \mathrm{~mm}$ valve with a higher outer sealing skirt than smaller sized valves [2-4]. It has led to an increase in height of the sealing skirt by $40 \%$ in S3 Ultra valves resulting in a $50 \%$ increase of the area of contact with the native anatomy. The sealing PET skirt has a textured structure which promotes healing and endothelialization. The external skirt design is introduced in 20, 23 and $26 \mathrm{~mm}$ S3 Ultra valves while the $29 \mathrm{~mm}$ valve remains the current S3 model.

\section{The delivery system}

New features of the S3 Ultra delivery system reduce the number of procedural steps and offers low system profiles across all valve sizes. The S3 Ultra valve is crimped directly on the balloon, so there is no need to align it in the descending aorta nor to retract the flex catheter before implantation. Also, the distal tip of the balloon was redesigned: it has a short, tapered tip with a smooth transition to the valve and a lower crossing profile. The delivery catheter has controlled dual articulation which allows avoiding contact with aortic walls during crossing of the arch.

\section{The delivery sheath}

The Axela sheath is an expandable, and selfcollapsible $14 \mathrm{~F}$ system with a hydrophilic coating. The expansion of the sheath is transient as it has an outer elastomeric jacket which forces active contraction of the sheath walls after valve passage. The $14 \mathrm{~F}$ sheath is compatible with all valve sizes. It has a seamless design to reduce the risk of bleeding or vessel trauma during the procedure.

For the transapical approach, the Certitude delivery system allows the use of S3 Ultra valves

Address for correspondence: Wojciech Wojakowski, MD, PhD, Division of Cardiology and Structural Heart Diseases, Medical University of Silesia, ul. Ziolowa 45, 40-635 Katowice, Poland, e-mail: wwojakowski@sum.edu.pl

Received: 10.01.2019

Accepted: 17.02.2019

*Contributed equally. 


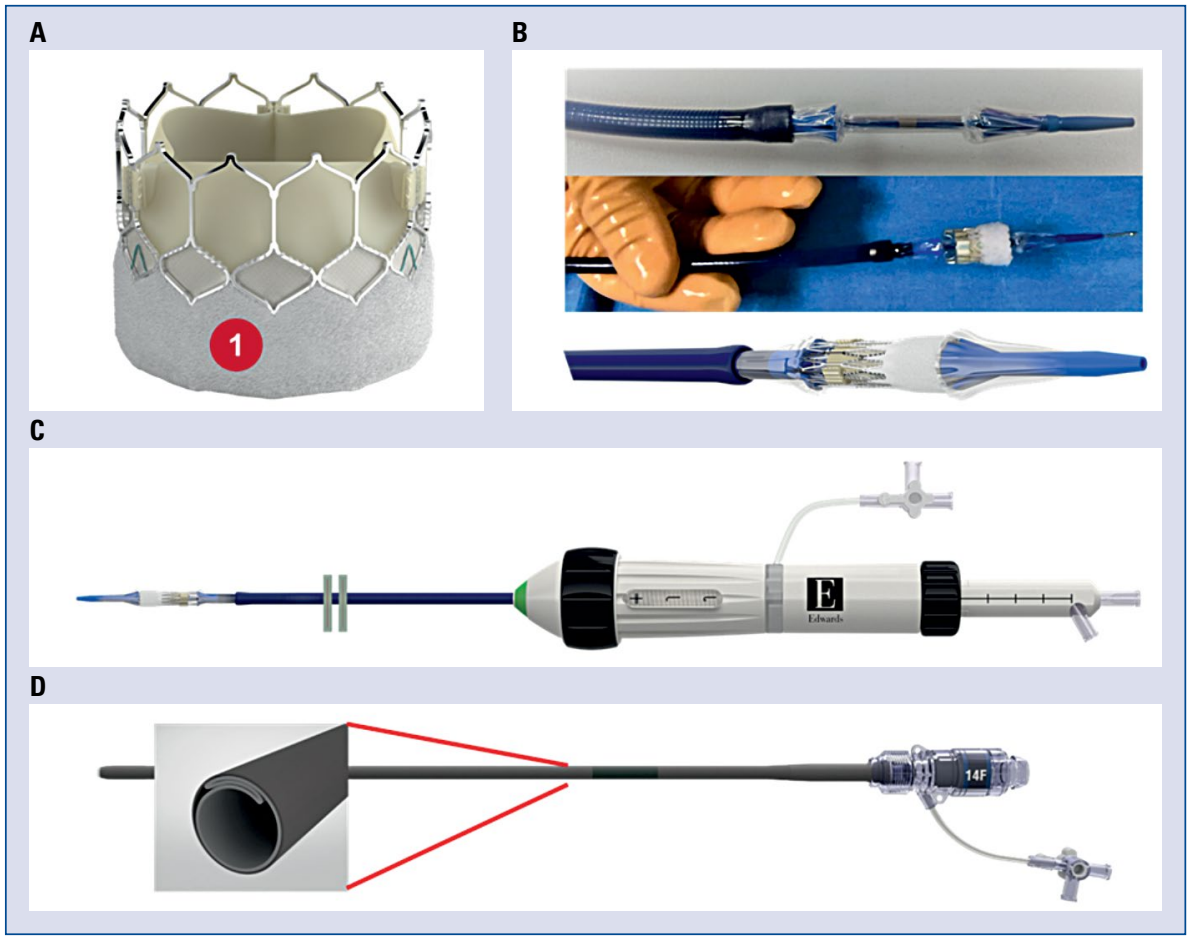

Figure 1. The SAPIEN 3 Ultra system; A. S3 Ultra valve with a redesigned outer skirt (1); B. Balloon and a crimped valve; C. Delivery system; D. Axela sheath with a cross-section showing an outer elastomeric jacket and a folded expandable wall of the inner sheath. Modified from images provided by Edwards Lifesciences with written permission to use in print.

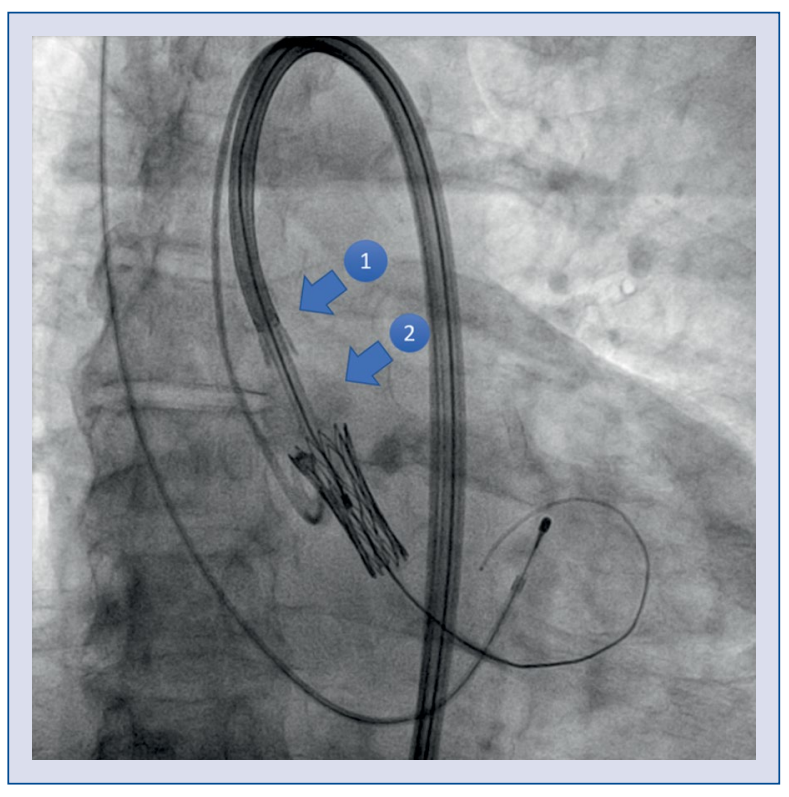

Figure 2. The SAPIEN 3 Ultra implantation; 1 - The fluoroscopic appearance of the flex catheter without a retractable pusher; 2 - Inflation of the balloon starting at its proximal end.

with $18 \mathrm{~F}$ sheaths for $20 \mathrm{~mm}$ or $21 \mathrm{~F}$ sheaths for 23-29 mm valves.

\section{Procedural remarks}

The recommendations for valve sizing based on the angio-computed tomography remain consistent with S3. The differences during the procedure are mainly related to tracking and positioning of the new delivery system and the valve. Since there is no need for valve alignment in the descending aorta, the new delivery system can be flexed earlier than the S3 Commander system to avoid contact with the aortic wall. The S3 Ultra can be positioned for implantation directly after crossing of the native valve. It is recommended to push the valve into the left ventricle slightly lower than the intended level of implantation, then withdraw it and by using the distal handle knob to fine-tune the optimal position. Also, it is suggested to place the radiopaque marker 1-2 $\mathrm{mm}$ higher above the annular plane than for the S3 valve. Importantly, the expansion of the newly designed balloon starts at the proximal (aortic) end, unlike in the S3 system (Fig. 2). Therefore, it is not possible with S3 Ultra to use a small amount of contrast to dilate the distal end of the balloon to facilitate the direct implantation in case of difficult crossing of a calcified native valve. Finally, the balloon should be fully emptied from contrast before its withdrawal into the Axela sheath. 
The initial experience of five procedures using the S3 Ultra in patients with severe stenosis of tricuspid and bicuspid native aortic valves and for a valve-in-valve implantation showed easy access site handling, good trackability and procedural results with no PVL.

Conflict of interest: Radosław Parma, Wojciech Wojakowski - Lecture honoraria: Edwards Lifesciences.

\section{References}

1. Solomonica A, Choudhury T, Bagur R. Newer-generation of Edwards transcatheter aortic valve systems: SAPIEN 3, Centera, and SAPIEN 3 Ultra. Expert Rev Med Devices. 2019; 16(2): 81-87, doi: 10.1080/17434440.2019.1555465, indexed in Pubmed: 30501446.

2. Daubert MA, Weissman NJ, Hahn RT, et al. Long-Term Valve Performance of TAVR and SAVR: A Report From the PARTNER I Trial. JACC Cardiovasc Imaging. 2016 [Epub ahead of print], doi: 10.1016/j.jcmg.2016.11.004, indexed in Pubmed: 28017714.

3. Kapadia SR, Leon MB, Makkar RR, et al. PARTNER trial investigators. 5-year outcomes of transcatheter aortic valve replacement compared with standard treatment for patients with inoperable aortic stenosis (PARTNER 1): a randomised controlled trial. Lancet. 2015; 385(9986): 2485-2491, doi: 10.1016/S01406736(15)60290-2, indexed in Pubmed: 25788231.

4. Hahn RT, Pibarot P, Leipsic J, et al. The Effect of Post-Dilatation on Outcomes in the PARTNER 2 SAPIEN 3 Registry. JACC Cardiovasc Interv. 2018; 11(17): 1710-1718, doi: 10.1016/j. jcin.2018.05.035, indexed in Pubmed: 30121276. 